\title{
New Algorithms to Map Asymmetries of 3D Surfaces
}

\author{
Benoît Combès and Sylvain Prima \\ INSERM, U746, F-35042 Rennes, France \\ INRIA, VisAGeS Project-Team, F-35042 Rennes, France \\ University of Rennes I, CNRS, UMR 6074, IRISA, F-35042 Rennes, France \\ \{bcombes, sprima\}@irisa.fr \\ http://www.irisa.fr/visages
}

\begin{abstract}
In this paper, we propose a set of new generic automated processing tools to characterise the local asymmetries of anatomical structures (represented by surfaces) at an individual level, and within/between populations. The building bricks of this toolbox are: 1) a new algorithm for robust, accurate, and fast estimation of the symmetry plane of grossly symmetrical surfaces, and 2) a new algorithm for the fast, dense, nonlinear matching of surfaces. This last algorithm is used both to compute dense individual asymmetry maps on surfaces, and to register these maps to a common template for population studies. We show these two algorithms to be mathematically well-grounded, and provide some validation experiments. Then we propose a pipeline for the statistical evaluation of local asymmetries within and between populations. Finally we present some results on real data.
\end{abstract}

\section{Introduction}

Improved characterisation of asymmetries should help understanding the brain in normal and pathological conditions. The development of techniques for 3D imaging (especially MRI), and of processing tools for the automated analysis of grey level images has allowed the systematic study of these asymmetries [56]. However, artefacts in MR images, such as noise and intensity inhomogeneities, have severe confounding effects on subsequent asymmetry analyses. An alternative approach to characterise asymmetries is to segment the structure of interest first (using manual/semi-automated/automated techniques dealing with image artefacts), and then to analyse the segmented structure, by studying its boundary surface.

To our knowledge, there are only few methods allowing the automated pointwise mapping of surface asymmetries. A first approach consists in registering a template, perfectly symmetrical surface, to the surface of interest in a nonlinear way. The difference between the two displacement vectors needed to map bilateral points of the template with points of the surface is used to quantify the local asymmetry at these points [7. A second approach normalises the surface of interest in a reference frame using several manually selected bilateral

D. Metaxas et al. (Eds.): MICCAI 2008, Part I, LNCS 5241, pp. 17 25, 2008.

(C) Springer-Verlag Berlin Heidelberg 2008 
landmarks. In this reference frame, using a cylindrical coordinate system, the residual difference of radial coordinates between points of the surface having identical height and opposite azimuthal coordinates is used to quantify the local asymmetry at these points [8]. In our opinion, these methods suffer from two main limitations. First, they assume that a template (in the first approach) or a reference coordinate system (in the second) are available, which limits their application to specific problems. Second, and most importantly, the resulting mappings are extrinsic, as they are based on these precomputed data, and thus potentially biased by the chosen reference system.

In this paper, we propose a set of new generic automated processing tools to characterise the local asymmetries of anatomical structures (represented by surfaces) at an individual level, and within/between populations. The building bricks of this toolbox are: 1) a new algorithm for robust, accurate, and fast estimation of the symmetry plane of grossly symmetrical surfaces (Section 2), and 2) a new algorithm for the dense, non-linear matching of surfaces (Section 3). This last algorithm is used both to compute dense individual intrinsic asymmetry maps on surfaces, and to register these maps to a common template for population studies. We show these two algorithms to be mathematically well-grounded, and provide some validation experiments. Then we propose a pipeline for the statistical evaluation of local asymmetries within and between populations (Section 4). Finally we present some results on real data: computation of individual asymmetry maps on brain cortex, caudate nuclei and ventricles, and statistical comparison of facial asymmetry between males and females (Section 5). We conclude and give some perspectives in Section 6 .

\section{Symmetry Plane Computation as a ML Estimation}

In this section the surface under study is represented by a cloud of points noted $O$. For an ideal bilateral surface having a perfect symmetry, there exists a plane $P$ superposing each point $x$ with its counterpart in the other side of the surface, which writes $O=S_{P}(O)$. However, we only deal with grossly symmetrical surfaces and thus, the cloud $S_{P}(O)$ is viewed as a noised version of $O$. In practice, we define the probability function of the data points $y_{j} \in S_{P}(O)$ as a mixture density (dependent on the unknown plane $P$ ) using the points $x_{i} \in O$ as follows:

$$
p\left(y_{j} ; P\right)=\sum_{x_{i} \in O} p\left(y_{j}, x_{i} ; P\right)=\sum_{x_{i} \in O} A_{i, j} p\left(y_{j} \mid x_{i} ; P\right)
$$

There are as many mixture components as there are points in $O$. Intuitively, the unknown mixture component $A_{i, j}$ conveys the affinity between the points $y_{j}$ and $x_{i}$. Note that $\sum_{i} A_{i, j}=1$. If we consider all the data points $y_{j}$ to be independent, then the likelihood of the cloud $S_{P}(O)$ can be written as:

$$
L(P)=\prod_{y_{j} \in S_{P}(O)} \sum_{x_{i} \in O} A_{i, j} p\left(y_{j} \mid x_{i} ; P\right)
$$


We then define the optimal plane $\tilde{P}$ using the maximum likelihood principle. Considering the conditional densities $p\left(y_{j} \mid x_{i} ; P\right)$ as Gaussian with variance $\sigma^{2}$, we use the Expectation-Maximisation (EM) algorithm to estimate $P$. If we note $x_{j}=S_{P}\left(y_{j}\right) \in O$ for the sake of simplicity, the EM algorithm can be shown to yield the very simple following iterative scheme:

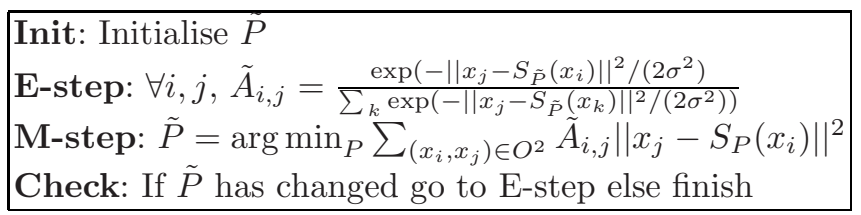

The M-step has a closed-form solution and the algorithm can be shown to converge to a local maximum of the likelihood function. Further implementation choices, details on how to deal with outliers, and complete evaluation of the algorithm can be found elsewhere [9].

\section{Dense Non-linear Matching as a MAP Estimation}

Numerous algorithms have been proposed to estimate non-linear transformations between 3D surfaces. One of the most successful techniques 11 to have been proposed for this purpose was inspired by the ICP algorithm [10]. It consists in 1) defining a spherical neighbourhood around each point of the moving surface, 2) matching each point within this neighbourhood with its closest point on the reference surface, 3) estimating the optimal rigid-body transformation using these correspondences, and 4) smoothing this set of pointwise rigid-body transformations to get locally affine transformations. This algorithm suffers from two major problems: 1) there is no proof of its convergence, and 2) the linear part $(3 \times 3$ matrix $)$ of the final pointwise affine transformation is computed by weighted averaging of the rotation matrices "entrywise", which is not optimal.

In the following, we propose a new algorithm to deal with these two problems. As Feldmar \& Ayache, we estimate one affine transformation for each point of the moving surface, but without using its neighbours. Instead, we add a spatial constraint on the estimated transformation, so that it is close to the transformations estimated at neighbouring points. This is performed using a probabilistic interpretation of the dense non-linear matching, which is viewed as a maximum a posteriori (MAP) estimation problem.

We consider two surfaces $X=\left\{x_{i}, i=1, \ldots, L\right\}$ and $Z=\left\{z_{k}, k=1, \ldots, M\right\}$. The problem is 1) to match each point $x_{i}$ in $X$ with a point $y_{i}=z_{j}$ in $Z$, and 2) to compute the affine transformation $T_{i}$ relating $x_{i}$ with $y_{i}$. Let us name $Y=\left\{y_{i}, i=1, \ldots, L\right\}$ and $T=\left\{T_{i}, i=1, \ldots, L\right\}$. We consider each point $x_{i} \in X$ (resp. each $T_{i} \in T$ ) as the realisation of a random variable $\mathbf{X}_{\mathbf{i}}$ (resp. $\mathbf{T}_{\mathbf{i}}$ ). We name $\mathbf{X}$ and $\mathbf{T}$ the two related sets of random variables. Then we suppose that $\mathbf{X}_{\mathbf{i}}, \mathbf{T}_{\mathbf{i}}$ and $y_{i}$ relate to each other following: $\mathbf{X}_{\mathbf{i}}=\mathbf{T}_{\mathbf{i}}\left(y_{i}\right)+\epsilon$, where $\epsilon \sim \mathcal{N}\left(0, \sigma^{2}\right)$ as in Section 2. If we assume that the random variables $\mathbf{X}_{\mathbf{i}}$ are independent 
then we can write the conditional probability of $\mathbf{X}$ given $\mathbf{T}$ (which is also the likelihood of the data $X$ ) as:

$$
l(\mathbf{X}=X \mid \mathbf{T}=T ; Y)=\frac{1}{(2 \pi)^{3 L / 2} \sigma^{3 L}} \exp \sum_{i=1, \ldots, L}-\frac{\left\|x_{i}-T_{i}\left(y_{i}\right)\right\|^{2}}{2 \sigma^{2}}
$$

It is not possible to compute the transformations $T_{i}$ in a unique way without additional constraints. Given that neighbouring points must have similar $T_{i}$, we consider that $\mathbf{T}$ is a Markov random field, with a Gibbs p.d.f. defined as $\left(A_{i}\right.$ and $t_{i}$ being the linear and translation components of $T_{i}$ ):

$$
P(\mathbf{T}=T)=\frac{1}{Z} \exp \left(-\sum_{(i, j) \in C_{2}(X)}\left(\alpha\left\|A_{i}-A_{j}\right\|_{F}^{2}+\beta\left\|t_{i}-t_{j}\right\|_{F}^{2}\right)\right)
$$

where $Z$ is a normalisation constant, \|\|$_{F}$ is the Frobenius norm, $\alpha, \beta$ are positive numbers, and $C_{2}(X)$ is the set of second-order cliques of $X$. In practice, the cliques are determined using a mesh: $(i, j) \in C_{2} \Leftrightarrow$ there exists an edge between points $x_{i}$ and $x_{j}$. Following Bayes rule, we then have $P(\mathbf{T} \mid \mathbf{X}) \alpha l(\mathbf{X} \mid \mathbf{T} ; Y) P(\mathbf{T})$.

The optimal set of transformations $T=\left\{T_{i}, i \in 1, \ldots, L\right\}$ is then defined as the one maximising this posterior probability. This optimisation can be performed using the ICM algorithm [12, which consists in considering sequentially each $T_{i} \in T$ and maximising the posterior for $T_{i}$ while keeping the other transformations fixed. This algorithm converges monotonically to a (at least local) maximum of the criterion, and boils down to:

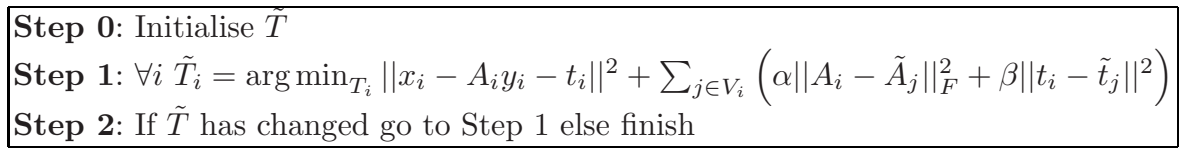

where $V_{i}$ contains the indices of neighbours of point $x_{i}$ in $X$. It can be shown that Step 1 has a unique solution (provided $\alpha>0$ and $\beta \geq 0$ ) that is given by the following closed form solution (with $N_{i}=\operatorname{card}\left(V_{i}\right)$ ):

$$
\left\{\begin{array}{l}
\tilde{A}_{i}=\left[\frac{\beta}{1+\beta N_{i}}\left(N_{i} x_{i}-\sum_{j \in V_{i}} \tilde{t}_{j}\right) y_{i}^{T}+\alpha \sum_{j \in V_{i}} \tilde{A}_{j}\right]\left[\frac{\beta N_{i}}{1+\beta N_{i}} y_{i} y_{i}^{T}+\alpha N_{i} I_{3}\right]^{-1} \\
\tilde{t}_{i}=\frac{1}{1+\beta N_{i}}\left(x_{i}-\tilde{A}_{i} y_{i}+\beta \sum_{j \in V_{i}} \tilde{t}_{j}\right)
\end{array}\right.
$$

This solution assumes that the set of correspondences $Y$ is known, which is not the case. In practice, the set $Y$ can be seen as a parameter of the conditional p.d.f. of $\mathbf{X} \mid \mathbf{T}$, and can be estimated within the ICM using the ML principle, which yields the following final iterative algorithm, that can also be shown to converge monotonically to a local minimum of $P(\mathbf{T} \mid \mathbf{X})$ :

Step 0: Initialise $\tilde{T}$
Step 1: $\tilde{Y}=\arg \max _{Y} l(\mathbf{X}=X \mid \mathbf{T}=\tilde{T} ; Y)$
Step 2: $\tilde{T}=\arg \max _{T} P(\mathbf{T}=T \mid \mathbf{X}=X ; \tilde{Y})$
Step 3: If $\tilde{T}$ has changed go to Step 1 else finish


Step 1 is simply solved by: $\tilde{Y}=\left\{\tilde{y}_{i}=\arg \min _{z_{k} \in Z}\left\|x_{i}-\tilde{T}_{i}\left(z_{k}\right)\right\|\right.$ for $i=$ $1, \ldots, L\}$. Step 2 is solved thanks to the iterative algorithm previously described. The overall algorithm has a very intuitive interpretation, as it simply boils down to the iterative estimation of the optimal correspondences between the two surfaces and the local affine transformations best superposing the two surfaces given the correspondences. In practice, the initial $\tilde{T}$ is chosen as the similarity transformation best superposing $X$ and $Z$ [10. Moreover, we tackle outliers at the end of Step 1 by detecting points $x_{i}$ for which $y_{i}$ is a border point of $Z$ or has a normal too different from that at $x_{i}$. The data attachment term is then set to 0 in Step 2 for these terms, whose $\tilde{T}_{i}$ is then estimated using only the regularisation term. A simpler, degenerate model, consists in choosing $T_{i}$ as a pure translation. The comparative merits of the two models are still to be evaluated.

Note that the proposed algorithm is close to the one recently proposed by Amberg et al. [13. However, our Markovian interpretation allows to solve Step 2 with the ICM algorithm. This provides advantages in terms of computational time and implementation easiness compared to the solution proposed by Amberg et al., which implies inversion of a sparse square matrix of size $(4 \operatorname{card}(X))^{2}$. The same can be said about many other competing algorithms, for instance those using thin plane splines as a regulariser [1415 which are inadequate for many applications with large point sets. On the contrary, our algorithm has an average run time of 4 min on a standard PC for surfaces with 100,000 points.

\section{Asymmetry Mapping}

\subsection{Pipeline}

Using the tools presented in Sections 2 and 3 . we propose the following pipeline for statistical analysis of asymmetry on surfaces $X_{1}, \ldots, X_{n}$ :

- Step 1: Estimation of the symmetry plane $P_{k}$ of $X_{k} \forall k=1, \ldots, n$

- Step 2: Non-linear registration of $X_{k}$ with $S_{P_{k}}\left(X_{k}\right)$ : each point $x_{i}$ in $X_{k}$ is matched with a point $y_{i}$ in $S_{P_{k}}\left(X_{k}\right) ; x_{i}-y_{i}$ quantifies the asymmetry at point $x_{i}$ (individual asymmetry mapping)

- Step 3: Non-linear registration of $X_{k}$ with a template surface $T$ : each point $t_{i}$ in $T$ is matched with a point $x_{i}$ in $X_{k}$; the asymmetry measure at point $x_{i}$ is then projected to point $t_{i}$ on the template (normalised asymmetry mapping)

- Step 4: Statistical tests (within/between populations) at each point of $T(t$ test, Hotelling's test, etc.) with/without correction for multiple comparisons.

Steps 1-2-3 are displayed on Fig. 1. Once the vector $x_{i}-y_{i}$ is computed, it is possible to compute its signed (or unsigned) norm which makes the projection on the template easier and the display visually appealing. The sign is computed using the dot product between $x_{i}-y_{i}$ and the normal at $x_{i}$ (computed using a mesh for instance). The template $T$ is chosen as one of the images in the dataset and made symmetrical after its symmetry plane is estimated. 


\subsection{Validation of the Individual and Normalised Mappings}

We evaluate the accuracy of the non-linear registration for individual and normalised asymmetry mapping on synthetic and real data. The parameters of the two algorithms (symmetry plane estimation and registration) are $\sigma=0.5$ and $\alpha=\beta=20$ (initial value) to 0.5 (final value) using a decreasing scale factor of 1.2. We consider 15 surfaces (laser scans of human faces). We manually define 10 homologous landmarks on each surface. These landmarks are chosen to be either pairs of bilateral or mid-facial points. Three experiments are devised:

- We register the 15 surfaces to the template $T$, compute the mean error between the homologous landmarks, and average these errors over the 15 surfaces.

- We compute the symmetry plane of each surface, flip the surface with respect to the plane, register the two surfaces, compute the mean error between the homologous landmarks, and average these errors over the 15 surfaces.

- We choose one of the 15 surfaces, make it symmetrical after computing its symmetry plane, add unilateral deformations and remove a given quantity of adjacent points (to generate occlusions). We map the unsigned asymmetries of the surface and project them to the template (Fig. 2). We also map the asymmetries of the surface using the approach of Ólafsdóttir et al. (performing the registration task with our tool for fair comparison) [7.

The conclusions are twofold. First, the mean error in the first experiment $(2.63 \mathrm{~mm} \pm 0.59 \mathrm{~mm})$ is larger than the error in the second $(0.67 \mathrm{~mm} \pm 0.13 \mathrm{~mm})$,
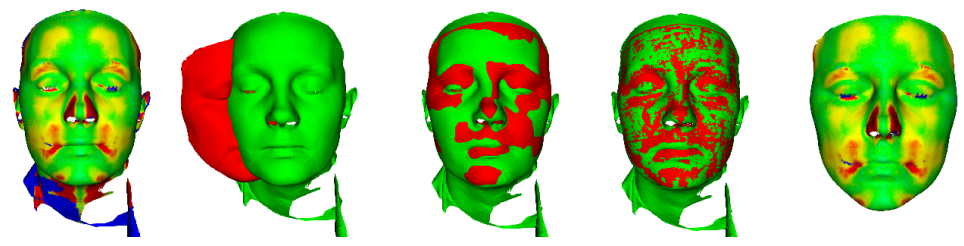

Fig. 1. Individual and normalised asymmetry mapping. From left to right: individual asymmetry mapping; surface and template before registration; surface and template after similarity-based registration; surface and template after non-linear registration; normalised asymmetry mapping on the template.
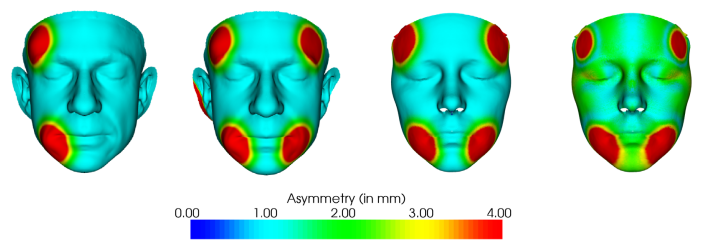

Fig. 2. Evaluation of the asymmetry mapping / comparison with another method. From left to right: a deformation field is applied to a perfectly symmetrical face; estimated asymmetry map by our method; normalised asymmetry map on the template; template-based asymmetry map as estimated by another method [7. 
which is intuitive and simply suggests that two halves of a given face are more similar than two different faces. It must be noted that the measured errors include the imprecision of the manual delineation of the landmarks. Second, our algorithm has a mean accuracy of $0.6 \mathrm{~mm} \pm 0.5 \mathrm{~mm}$ when recovering ground truth asymmetries, and proves to be superior to the competitive algorithm [7]. This is not surprising, as in this last method the individual asymmetry mapping is based on the registration of the two sides of the surface with the template, which is less precise than registering the two sides of the same surface together.

\section{Results}

We illustrate our method on different applications. First, we segment the brain cortex (300,000 points, brainvisa.info), the caudate nuclei and the ventricles (10,000 points, itksnap.org) from the T1-weighted MRI of a healthy subject, and we compute individual asymmetry maps (Fig. 3). Second, we perform the statistical analysis of face asymmetry within and between 15 healthy males and 15 females (Fig. 4) using laser scans (80,000 points, fastscan3d.com).

Interestingly, we recover the well-known Yakovlevian torque of the cortex (opposite asymmetry of the occipital and frontal lobes), hypothesized to be linked to language. Registering the two hemispheres is a difficult task and other non-linear techniques [16] will be tested for comparison with ours in future works. Mapping asymmetry of caudate nuclei is also of interest, as e.g. anomalies of left-right volume differences have been shown to predict attention-deficit hyperactivity disorders in children [17. Similarly, asymmetry of lateral ventricles in utero has
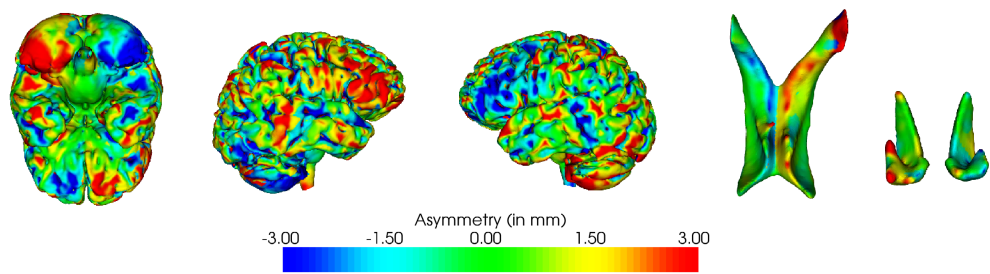

Fig. 3. Individual asymmetry mapping on a given subject. Left to right: brain cortex, lateral ventricles and caudate nuclei. Different views.
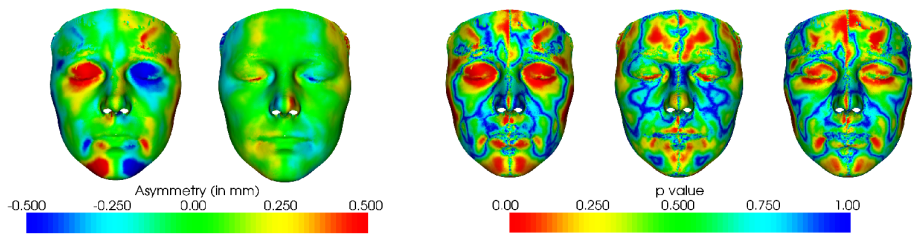

Fig. 4. Statistical analysis of asymmetry. Left to right: mean signed asymmetry maps on men/women, uncorrected p-value maps (using $t$-tests) of statistically significant asymmetry among men, among women, and comparison between men and women. 
been hypothesized to be indicative of cerebral pathology [18. At last, preliminary results on facial data suggest that the women are more symmetrical than men. Recent works also suggest that subtle facial dysmorphologies/asymmetries, could be present in schizophrenia [19].

\section{Conclusion}

We have presented a set of tools for the pointwise asymmetry mapping of 3D surfaces and statistical analysis of these asymmetries within and between populations. We have proved our methods to be theoretically well-grounded, provided some validation experiments, and shown them to be superior to a competitive state-of-the-art method. Further work will consist in testing other operators on the asymmetry vector field to emphasize other types of asymmetries (e.g. shrinking/expanding regions compared to contralateral areas). Comparisons with other techniques [6] based on grey value images will also be performed.

\section{References}

1. Toga, A., Thompson, P.: Mapping brain asymmetry. Nature Reviews Neuroscience 4(1), 37-48 (2003)

2. Holloway, R.L., et al.: Brain endocast asymmetry in pongids and hominids: some preliminary findings on the paleontology of cerebral dominance. Am. J. Phys. Anthropol. 58(1), 101-110 (1982)

3. Gannon, P.J., et al.: Asymmetry of chimpanzee planum temporale: humanlike pattern of Wernicke's brain language area homolog. Science 279(5348), 220-222 (1998)

4. Crow, T.: Cerebral asymmetry and the lateralization of language: core deficits in schizophrenia as pointers to the genetic predisposition. Current Opinion in Psychiatry 17(2), 97-106 (2004)

5. Good, C., et al.: Cerebral asymmetry and the effects of sex and handedness on brain structure: a voxel-based morphometric analysis of 465 normal adult human brains. NeuroImage 14(3), 685-700 (2001)

6. Thirion, J.-P., et al.: Statistical analysis of normal and abnormal dissymmetry in volumetric medical images. Medical Image Analysis 4(2), 111-121 (2000)

7. Ólafsdóttir, H., et al.: A point-wise quantification of asymmetry using deformation fields: application to the study of the Crouzon mouse model. In: Ayache, N., Ourselin, S., Maeder, A. (eds.) MICCAI 2007, Part II. LNCS, vol. 4792, pp. 452 459. Springer, Heidelberg (2007)

8. Liu, Y., Palmer, J.: A quantified study of facial asymmetry in 3D faces. In: IEEE Workshop on Analysis and Modeling of Faces and Gestures, pp. 222-229 (October 2003)

9. Combès, B., et al.: Automatic symmetry plane estimation of bilateral objects in point clouds. In: IEEE CVPR (June 2008)

10. Besl, P., McKay, N.: A method for registration of 3-D shapes. IEEE PAMI 14(2), 239-256 (1992)

11. Feldmar, J., Ayache, N.: Rigid, affine and locally affine registration of free-form surfaces. Int. J. Comput. Vision 18(2), 99-119 (1996)

12. Besag, J.: On the statistical analysis of dirty pictures. Journal of the Royal Statistical Society B-48(3), 259-302 (1986) 
13. Amberg, B., et al.: Optimal step nonrigid ICP algorithms for surface registration. In: IEEE CVPR, pp. 1-8 (June 2007)

14. Chui, H., Rangarajan, A.: A new point matching algorithm for non-rigid registration. Computer Vision and Image Understanding 89(2-3), 114-141 (2003)

15. Jian, B., Vemuri, B.: A robust algorithm for point set registration using mixture of Gaussians. In: IEEE ICCV, pp. 1246-1251 (October 2005)

16. Bansal, et al.: ROC-based assessments of $3 \mathrm{D}$ cortical surface-matching algorithms. NeuroImage 24(1), 150-162 (2002)

17. Achiron, R., et al.: Cerebral lateral ventricular asymmetry: is this a normal ultrasonographic finding in the fetal brain? Obstet. Gynecol. 89(2), 233-237 (1997)

18. Castellanos, F.X., et al.: Quantitative morphology of the caudate nucleus in attention deficit hyperactivity disorder. Am. J. Psychiatry 151(12), 1791-1796 (1994)

19. Hennessy, R., et al.: Facial shape and asymmetry by three-dimensional laser surface scanning covary with cognition in a sexually dimorphic manner. J. Neuropsychiatry Clin. Neurosci. 18(1), 73-80 (2006) 